\title{
6 Digitale Transformation durch elearning
}

\author{
Bernhard Breil
}

\section{Hintergrund}

Die Digitale Agenda der Bundesregierung, die Förderinitiative „Medizininformatik“ des Bundesministeriums für Bildung und Forschung (BMBF) sowie das 2015 verabschiedete eHealth-Gesetz sind nur einige Initiativen, um die Digitalisierung im Gesundheitswesen voran zu bringen. So unterschiedlich die Ansätze auch sind ist in allen Bereichen ein dringender Aus- und Weiterbildungsbedarf für die Berufstätigen im Gesundheitswesen erkennbar. Dieser Bedarf zieht sich durch alle Ebenen und ist sowohl in den Heilberufen (Ärzte und Pflegekräfte), in den technischen Berufen (Informatiker, Techniker) als auch auf der Management- und Führungsebene relevant.

Denn mit zunehmender Digitalisierung erhöht sich der Wissenszuwachs in der Medizin und Informatik weiter. Das Themenfeld Medizinische Informatik ist hierbei von besonderer Bedeutung, treffen mit der Medizin und Informatik zwei Fächer aufeinander, die vom stetigen Wissenszuwachs und zahlreichen Veränderungen durch neue Technologien (Informatik) sowie vielen neuen Erkenntnissen aus der Forschung und klinischen Studien (Medizin) leben. Somit ist lebenslanges Lernen für die Beschäftigten im Gesundheitswesen dringend erforderlich. Die Digitalisierung ist in der Aus- und Weiterbildung jedoch nicht nur als Inhalt (digitale Patientenakte, elektronischer Austausch von Daten, Interoperabilität) von großer Bedeutung, sondern in der Lehre auch als Methode relevant. 


\section{Digitalisierung ist sowohl als Inhalt als auch als Methode von großer Bedeutung.}

Im Kontext der Digitalisierung des Gesundheitswesens muss daher über die Digitalisierung der Lehre gesprochen werden. Im Zeitalter des Internets bieten eLearningFormate die Chance, den stetigen Wissensbedarf an Themen rund um die Gesundheitsinformatik unabhängig von Raum und Zeit zu decken. Der berufsbegleitende Kompetenzerwerb für Zusatzqualifikationen zur Medizininformatik ist hier essenziell.

\section{elearning}

Bei der Digitalisierung muss also zwangsläufig über eLearning gesprochen werden. Kerres (2016) definiert hierzu:

Unter eLearning verstehen wir Lernangebote, bei denen digitale Medien (a) für die Präsentation und Distribution von Lerninhalten und/oder (b) zur Unterstützung zwischenmenschlicher Kommunikation zum Einsatz kommen.

Es geht also nicht zwingend darum, jeden Lerninhalt zu digitalisieren, sondern die Lernenden adäquat zu unterstützen. In einer Untersuchung des Deutschen Instituts für Erwachsenenbildung (DIE) und des Bundesinstituts für Berufsbildung (BIBB) kommen die Autoren zum Schluss, dass 2012 Angebote im Bereich eLearning/Fernlehrgänge vergleichsweise wenig verbreitet waren. eLearning tritt in verschiedenen Formen auf und reicht vom digitalen Informationsaustausch als Ergänzung zur Präsenzlehre über virtuelle Klassenzimmer, Webinare und Lernvideos bis zu komplexen Multimedia-Lerneinheiten, bei denen verschiedene Kanäle zu Informationsaufnahme adressiert werden. Dies basiert auf der Kognitiven Theorie des multimedialen Lernens nach Mayer (2005), nach der die Informationsaufnahme mittels zwei verschiedener Kanäle (visuell/bildhaft und auditiv/verbal) erfolgt. Weiterhin nimmt Mayer (2005) eine aktive Informationsverarbeitung an, was bedeutet, dass sich Lernende aktiv mit dem Lernmaterial beschäftigen, um eine kohärente mentale Repräsentation ihrer vorhandenen Erfahrungen konstruieren zu können. Beide Aspekte sind wichtige Grundlagen für das Lernen mit elektronischen Medien.

Mit Lernplattformen wie Moodle (www.moodle.de) und Ilias (http://www.ilias.de) stehen seit einiger Zeit entsprechende Werkzeuge zur Verfügung, verschiedene Formen von eLearning zu unterstützen. Neben Gruppensystemen für kooperatives Arbeiten, Test- und Bewertungswerkzeug bieten sie dem Nutzer durch Persönliche Ordner und Bookmarks, Glossar und Kalender sowie einem integrierten Wiki Möglichkeiten, um den eigenen Lernprozess zu steuern. Beide Plattformen unterstützen zudem mit den Standards SCROM 1.2 und SCORM 2004 ein Referenzmodell für austauschbare elektronische Lerninhalte. Sowohl Moodle als auch Ilias fallen unter die Kategorie Freie Software (GPL Lizenz). 


\section{Vor- und Nachteile von elearning}

eLearning ermöglicht einerseits ein zeit- und ortsunabhängiges selbstbestimmtes Lernen. Zum anderen erleichtert es die Aufnahme von Wissen durch multimediale und interaktive Elemente. Komplexe Abläufe und die Dynamik des Umfeldes können strukturiert und vereinfacht in verschiedenen Varianten dargestellt werden. Didaktisch gesteuerte Lernpfade führen den Lernenden durch das Material. Darüber hinaus lassen sich Lernkontrollen in Form von formativen Prüfungen unterschiedlicher Schwierigkeitsstufen einsetzen und eine kontinuierliche Überprüfung des eigenen Wissensstands zu ermöglichen. Weitere Vorteile ergeben sich durch ein eigenes Lerntempo.

\section{Der große Vorteil beim elearning ist das selbstbestimmte Lernen.}

Als Nachteil wird vor allem das geringe Angebot von qualifizierten Personen und Produkten gesehen. Zudem stellt sich die Frage nach Prüfungen. Welche Formate und Prüfungen werden von wem anerkannt? Wie kann bei Online-Prüfungen sichergestellt werden, dass die richtige Person am Computer sitzt? Zudem erfordern die Vorteile des selbstbestimmten Lernens ein bestimmtes Maß an Disziplin und Selbstlernkompetenz. Bei der eigenen Strukturierung der Lerninhalte droht eher Ablenkung oder ein Aufschiebeverhalten (Procrastination).

\section{eLearning-Angebote im Digitalen Gesundheitswesen}

Nach Reinmann (2010) nimmt die Institutionalisierung der Weiterbildung im Erwachsenalter ab. Dies ist Chance und Notwendigkeit zugleich, sich als Lernender entsprechende Angebote zu suchen. Diese Angebote kommen von einzelnen Personen, Organisationen oder von den Hochschulen. Letztgenannte haben längst erkannt, dass das Bildungsniveau fortlaufend aktualisiert und an neue Aufgaben und sich ändernde Bedingungen in Wirtschaft, Technologie und Recht angepasst werden muss. Die FernUniversität in Hagen setzt mit vielen akkreditierten Studiengängen seit Jahren auf das selbstbestimmte Lernen, wenngleich die Wissensvermittlung häufig über (digitale) Modulhandbücher und weniger über eLearning-Einheiten erfolgt.

\section{Bislang gibt es wenig elearning-Angebote im Digitalen Gesundheits- wesen.}

Sucht man nach modularen Angeboten im Gesundheitswesen, ist das Gesamtangebot noch überschaubar. Auf den Seiten der Deutschen Gesellschaft für Medizinische Informatik, Biometrie und Epidemiologie e.V. (GMDS) finden sich WeiterbildungsAngebote des Bundesverbands Gesundheits-IT (bvitg), des mibeg-Instituts für die Weiterbildungen in der Medizin, der Deutschen Informatik Akademie (DIA), des Deutschen Krankenhausinstituts (DKI) sowie Angebote privater Beratungsbüros wie 
beispielsweise eLearning4eHealth. Im Folgenden sollen einige dieser Angebote kurz skizziert werden, um die Bandbreite an verschiedenen Formen, Zielen und Inhalte deutlich zu machen.

Die DIA setzt bei ihren Kursen, die sich vor allem um IT-Themen ohne besonderen Bezug zum Gesundheitswesen drehen, auf Präsenzkurse und auch das DKI bietet den größten Teil der Weiterbildungsveranstaltungen als klassische Seminare an festen Orten an, setzen aber im Bereich Medizincontrolling auf Online-Trainings in Kooperation mit medcontroller (www.medcontroller.de). Während das mibeg-Institut in der ärztlichen Weiterbildung überwiegend auf Präsenzkurse setzt, haben andere Anbieter wie u.a. Thieme die Flexibilität von eLearning-Angeboten erkannt und vergeben CME-Punkte für das erfolgreiche Absolvieren von Online-Kursen, von denen mehr als 450 auf dem entsprechenden Portal bereitstehen (www.cme.thieme.de). Beim bvitg gibt es ein breites Angebot mit Fortbildungen in den Bereichen Medizininformatik, Standardisierung und Interoperabilität sowie Gesundheitswesen und Ordnungspolitik, von denen nun ein Teil als Webinare (wie bspw. Vergaberecht) angeboten wird. Orientiert an Inhalten vom Zertifikat Medizinische Informatik ermöglicht das Angebot eLearning4eHealth (www.elearning4ehealth.de) jedem Lernenden die Zusammenstellung der für ihn nutzbringenden Lerneinheiten, die wichtige Themen wie Medizinische Dokumentation, Informationssysteme im Gesundheitswesen (KIS, APIS), Management im Gesundheitswesen (Medizin- Controlling, QM) sowie Rahmenbedingungen (Ethik, Datenschutz, Risikomanagement) adressieren.

Das Zertifikat Medizinische Informatik ist ein von der GMDS, der Gesellschaft für Informatik (GI) sowie dem Berufsverband Medizinischer Informatiker (BVMI) gemeinschaftliche verliehenes Qualifikationszeugnis, das der Inhaberin oder dem Inhaber eine Qualifikation für verantwortliche Positionen in der Medizinischen Informatik hinsichtlich einer akademischen Ausbildung, einer fachlichen Fort- und Weiterbildung sowie einer 5-jährigen operationellen Tätigkeit in der Medizinischen Informatik bescheinigt. Informationen zum Zertifikat finden sich unter: https://gmds.de/ueber-uns/organisation/praesidiumskommissionen/ gmds-praesidiumskommission-zertifikat-medizinische-informatik-vorsitz-inf/.

\section{Ausblick}

Die Digitalisierung des Gesundheitswesens erfordert auch eine Digitalisierung der Lehrinhalte, denn damit gewinnt man die benötigte Flexibilität in der Weiterbildung. Hier gibt es erste Angebote, jedoch erfordert der wachsende Bedarf einen Ausbau, um den Anforderungen am Markt gerecht zu werden. Kritische Erfolgsfaktoren für erfolgreiche eLearning-Einheiten sind modulare, abgeschlossene Wissensbausteine von kurzer Dauer, die eine flexible Nutzung ermöglichen. Eine multimediale Unterstützung bietet durch Animationen zudem die Chance, mehrere Verarbeitungskanäle gleichzeitig anzusprechen. Darüber hinaus hängt der Erfolg von der Selbstdisziplin der Lernenden ab. Dies kann durch elektronische Tests für die Selbstkontrolle oder Betreuung unterstützt werden, aber der Lernende muss schlussendlich selbst mit der neuen Lernkultur zurechtkommen. Darüber hinaus wird es weiter notwendig sein, traditionelle Bildungsformen zu nutzen. 


\section{elearning kann auch im Zeitalter der Digitalisierung traditionelle Bil- dungsformen nicht ersetzen. Der größte Nutzen entsteht durch Kombi- nation beider Formate.}

Das Zusammenspiel mit traditionellen Lehrformen kann gelingen, wenn der Gesamtstoff in kleinere eLearning-Einheiten modularisiert werden kann. Für die Ersteller solcher eLearning Objekte (kurz eLOB) ist daher eine aufwändige Entwicklung und didaktisches Design für den Gesamtkurs sowie innerhalb der einzelnen eLOBs notwendig. Aufgrund des zuvor angesprochenen enormen Wissens- und Erkenntnisgewinns ist eine permanente Pflege unerlässlich.

\section{Literatur}

Ambos I, Koscheck S, Ohly H, Weiland M (2015) BIBB/DIE-wbmonitor 2013 - Lerndienstleistungen und neue Angebotsformen. (B. für Berufsbildung, Ed.) (GWA_1.0 ed.). Bonn: Forschungsdatenzentrum im BIBB

Kerres M (2016) Gestaltungsorientierte Bildungsforschung. URL: http://mediendidaktik.uni-due.de/lehrstuhlseite/5367 (letzter Aufruf 30.04.2017)

Mayer RE (2005) Cognitive theory of multimedia learning. In: Mayer RE (Hrsg.) The Cambridge Handbook of Multimedia Learning (S. 31-48). Cambridge, MA: Cambridge University Press

Reinmann G (2010) Bildungspsychologie des mittleren Erwachsenenalters. In: Spiel C, Schober B, Wagner P, Reimann R (Hrsg.) Bildungspsychologie. Hogrefe 